\title{
Graft-vs-Host Disease Colitis After Lung Transplant
}

\author{
Kavin Kanthasamy, MD${ }^{1}$, Mimi Tan Chang, MD², and Manreet Kaur, MD² \\ ${ }^{1}$ Division of Internal Medicine, John's Hopkins Hospital, Baltimore, MD \\ ${ }^{2}$ Section of Gastroenterology and Hepatology, Baylor College of Medicine, Houston, TX
}

\section{ABSTRACT}

Graft-vs-host disease, characteristically a major complication of allogenic hematopoietic stem cell transplantation, is rare after solid organ transplantation. We report a 50-year-old man who presented with abdominal pain, vomiting, and diarrhea shortly after bilateral lung transplantation. Colonoscopy with biopsy revealed diffuse severe active colitis with ulceration and crypt apoptosis consistent with graft-vs-host disease colitis. The diagnosis was confirmed by the presence of donor lymphocytes in the peripheral blood. His symptoms were refractory to corticosteroids but responded to the addition of infliximab and extracorporeal photophoresis. He remained in remission 17 months later.

\section{INTRODUCTION}

Graft-vs-host disease (GVHD) occurs when grafted immunocompetent donor T-lymphocytes attack host organs and classically manifests across the skin (rash), liver (cholestatic hepatitis), bone marrow (cytopenias), and gut (diarrhea). ${ }^{1}$ Solid organ transplant GVHD most commonly occurs in organs with large amounts of resident lymphoid tissue such as the small bowel and liver. ${ }^{2,3}$ Cases in organs with fewer lymphocytes, such as the lung, are especially rare and nearly always fatal. Of the 11 previous cases, 9 patients with lung GVHD died largely because of complications of pancytopenia and sepsis. ${ }^{4-8}$ We present a case of GVHD after lung transplantation with initially isolated gastrointestinal (GI) symptoms that was successfully treated.

\section{CASE REPORT}

A 50-year-old man, with gastroesophageal reflux disease, a remote history of peptic ulcer disease, and bilateral lung transplantation for pulmonary sarcoidosis 4 weeks before, presented with dull, mid-epigastric abdominal pain, 2 episodes of nonbilious, nonbloody emesis, and a 4-day history of diarrhea of 8-10 loose, maroon-colored stools per day. His active immunosuppression regimen consisted of prednisone $10 \mathrm{mg}$, mycophenolate $1,000 \mathrm{mg}$ BID, and tacrolimus $1.5 \mathrm{mg}$ BID. Other medications included voriconazole, valganciclovir, and trimethoprim/sulfamethoxazole for infection prophylaxis and a proton pump inhibitor 40 mg BID. He denied sick contacts, recent travel, and alcohol or tobacco use. The donor was cytomegalovirus (CMV) (+) and host was CMV ( - ). His vital signs were notable for a heart rate of 110 but were otherwise normal. On physical examination, he appeared fatigued and had diffuse epigastric tenderness to palpation without rebound or guarding hyperactive bowel sounds and no heptomegaly. The physical examination was otherwise normal. Liver function tests were notable for an aspartate aminotransferase of $101 \mathrm{U} / \mathrm{L}$, alanine aminotransferase of $269 \mathrm{U} / \mathrm{L}$, and albumin of $3.1 \mathrm{~g} / \mathrm{dL}$. Infectious workup including serum CMV immunoglobulin M/polymerase chain reaction (PCR), histoplasmosis, Coccidioides, Cryptosporidium, and Strongyloides antigen was negative. Stool Clostridium difficile PCR, stool culture, stool norovirus, enterovirus; adenovirus PCR, ova, and parasite examination; and fungal stains were negative. Colonoscopy revealed diffuse edematous, erythematous mucosa with overlying pseudomembranes from the cecum to the proximal transverse colon. The terminal ileum was denuded and erythematous with ulcerations and loss of villi (Figure 1).

Biopsy of the ascending colon showed marked crypt epithelial apoptosis consistent with GVHD colitis (Figure 2). No granulomas, viral inclusions (CMV, Herpes simplex viruses), fungi, or parasites were seen. The diagnosis of GVHD was confirmed with the presence of donor lymphocyte chimerism in the patient's peripheral blood. Intravenous methylprednisolone was started, and mycophenolate was discontinued, but the patient's diarrhea persisted after 10 days. He then developed a maculopapular rash with 


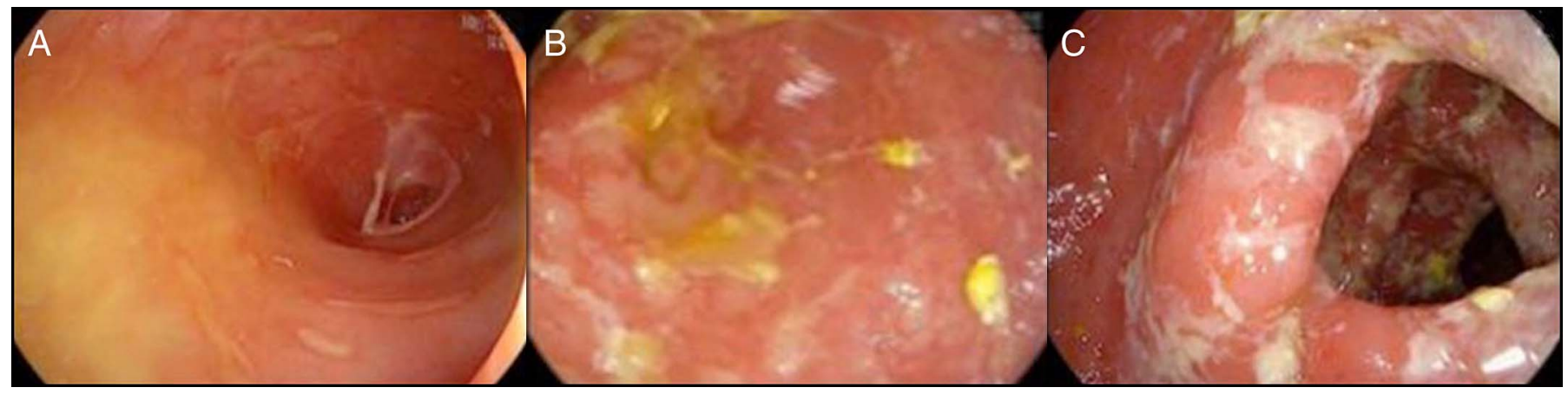

Figure 1. Colonoscopy images of the (A) terminal ileum, (B) cecum, and (C) transverse colon.

skin biopsy being consistent with GVHD. One dose of infliximab $5 \mathrm{mg} / \mathrm{kg}$ was given, and he was started on extracorporeal photophoresis (ECP) for steroid-resistant GVHD. His diarrhea ceased, rash resolved, and follow-up colonoscopy showed complete healing of colonic mucosa 3 months later (Figure 3). The initial transaminitis was attributed to hepatic GVHD and resolved after the dose of infliximab. He remained in remission 17 months later.

\section{DISCUSSION}

The pathogenesis of gut GVHD is thought to begin when preconditioning chemoradiation, underlying disease, or surgery inflicts a preliminary insult to host tissue. The resulting release of host proinflammatory cytokines (tumor necrosis factor- $\alpha$, interleukin-1, interleukin-6) upregulates host antigen-presenting cells and subsequently donor $\mathrm{T}$ cells to mediate mucosal damage. $^{1,4}$

GVHD most commonly involves the distal small bowel and colon. ${ }^{9}$ Secretory diarrhea is the predominant GI manifestation of acute GVHD, the volume of which may be used to grade disease severity and predict outcomes. ${ }^{9-11}$ Additional symptoms include anorexia, nausea/vomiting, abdominal pain, hematochezia, and protein malabsorption. Within the first 20 days of transplant, GVHD cannot be reliably distinguished from the effects of preconditioning chemoradiation and is considered between 20 and 100 days post-transplant. The clinical presentation of gut GVHD is nonspecific and requires histopathologic correlation on biopsy. Most of the studies support rectosigmoid biopsies, given their high sensitivity (82\%-95\%) regardless of the symptom profile. ${ }^{11,12}$ In hemopoeitc stem cell transplant patients with diarrhea, colonoscopy and sigmoidoscopy are equivalent in diagnosing GVHD, so the latter may benefit patients with a high procedural risk. ${ }^{12}$ Endoscopically, GVHD displays erythema, edema, erosions, and denudation of mucosa; however, approximately half of patients with normal colonoscopies and $60 \%$ with normal EGDs had histologic evidence of GVHD-strongly supporting obtaining biopsies irrespective of normal-appearing mucosa. ${ }^{13}$ Chimerism or the presence of donor lymphocytes in the patient's peripheral blood can confirm clinical suspicion of GVHD. Greater than $10 \%-20 \%$ chimersim has been shown to be highly specific for GVHD in liver transplantation. ${ }^{13}$

Mycophenolate mofetil (MMF), a common immunosuppressive drug used to lower the risk of graft rejection, causes colitis that clinically and pathologically resembles acute GVHD. Both share histologic features of crypt apoptosis; however, MMF enterocolitis can be differentiated by the presence of increased eosinophils ( $>15$ per 10 high-power field), the absence of endocrine cell aggregates within the lamina propria, and the lack of apoptotic microabscesses that are characteristic of GVHD. Crypt apoptosis combined with the lack of eosinophils and the persistence of symptoms after discontinuation of MMF favored GVHD colitis in the presented patient. ${ }^{14}$

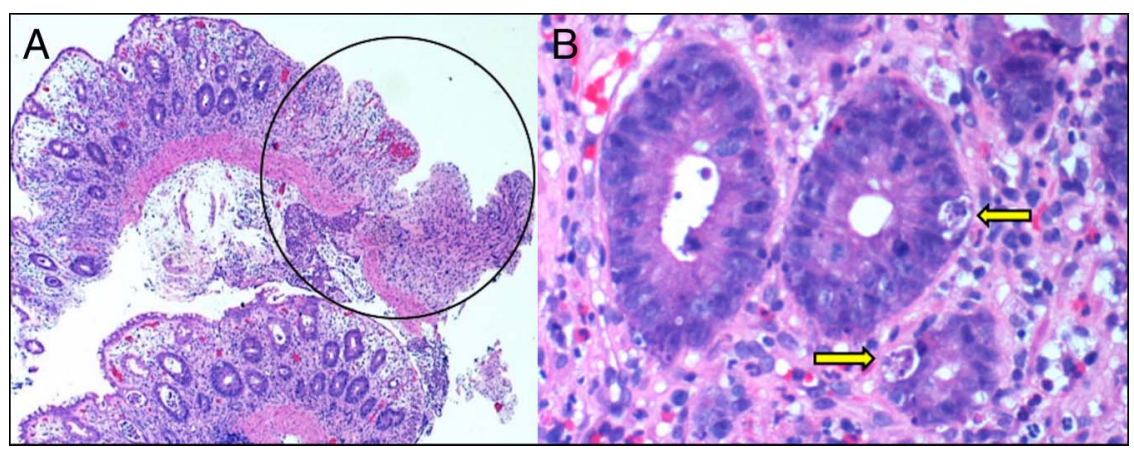

Figure 2. (A) Severe cryptitis with ulceration and crypt dropout (circle), and (B) apoptotic colonic epithelial cells aka "exploding crypt cells" (arrows). 


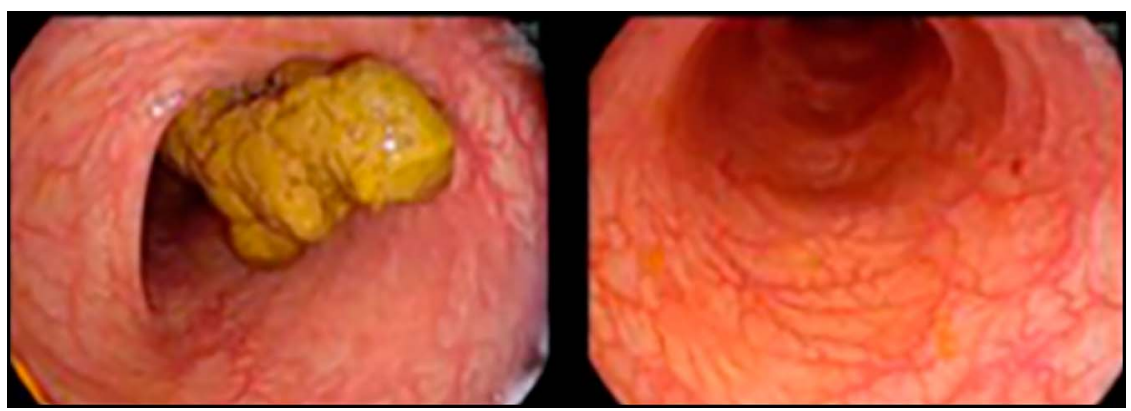

Figure 3. Follow-up colonoscopy 3 months after infliximab therapy showing complete healing of colonic mucosa.

Infectious mimics of GVHD, most notably CMV and Cryptosporidium, but also herpes simplex virus, adenovirus, Clostridium difficile, Campylobacter, and fungi, must be ruled out. There is a $0.2 \%$ incidence of de novo post-transplant inflammatory bowel disease, higher than that seen in the general population, and it is most commonly seen after liver transplant. ${ }^{15,16}$ There have been no cases of de novo inflammatory bowel disease reported post-lung transplant.

There is no standard treatment for GVHD, and approaches aim to suppress donor T-lymphocyte function initially with systemic corticosteroids. Budesonide increases treatment response for GI GVHD, and octreotide may control diarrheal volume. ${ }^{17}$ Infliximab has been shown to be effective in steroid-refractory GVHD, particularly in patients with GI involvement. Calcineruin inhibitors, MMF, monoclonal anti-IL2, and anti-CD3 have also been used. ECP provides a means to treat steroidrefractory GVHD without generalized immunosuppression via the reintroduction chemo-irradiated peripheral lymphocytes obtained through apheresis. ${ }^{18}$ The presented case appears to be the only known case of lung transplant GVHD successfully treated with ECP. Solid organ transplant GVHD is notoriously steroid refractory and requires expeditious diagnosis with prompt treatment to combat its poor prognosis.

\section{DISCLOSURES}

Author contributions: K. Kanthasamy wrote the manuscript and is the article guarantor. MT Chang and M. Kaur edited the manuscript.

Financial disclosure: None to report.

Informed consent was obtained for this case report.

Received July 26, 2019; Accepted October 7, 2019

\section{REFERENCES}

1. Ross WA, Couriel D. Colonic graft-versus-host disease. Curr Opin Gastroenterol. 2005;21:64-9.

2. Mazariegos GV, Abu-Elmagd K, Jaffe R, et al. Graft versus host disease in intestinal transplantation. Am J Transpl. 2004;4:1459-65.
3. Taylor AL, Gibbs P, Bradley JA. Acute graft versus host disease following liver transplantation: The enemy within. Am J Transpl. 2004;4:466-74.

4. Assi MA, Pulido JS, Peters SG, Mccannel CA, Razonable RR. Graft-vs.-host disease in lung and other solid organ transplant recipients. Clin Transpl. 2007;21(1):1-6.

5. Luckraz H, Zagolin M, McNeil K, Wallwork J. Graft-versus-host disease in lung transplantation: 4 case reports and literature review. J Heart Lung Transpl. 2003;22;691-7.

6. Smith DM, Agura ED, Ausloos K, Ring WS, Domiati-Saad R, Klintmalm GB. Graft-vs-host disease as a complication of lung transplantation. J Heart Lung Transpl. 2006;25:1175-7.

7. Worel N, Bojic A, Binder M, et al. Catastrophic graft-versus-host disease after lung transplantation proven by PCR-based chimerism analysis. Transpl Int. 2008;21:1098-101.

8. Pavenski K, Kamel-Reid S, Wei C, Cserti-Gazdewich CM. Lung transplantation complicated by graft-versus-host disease and confounded by incidental transfusion-associated macrochimerism. Transfusion. 2008;48: 2190-6.

9. Castilla-llorente C, Martin PJ, Mcdonald GB, et al. Prognostic factors and outcomes of severe gastrointestinal GVHD after allogeneic hematopoietic cell transplantation. Bone Marrow Transpl. 2014;49(7):966-71.

10. Ferrara JL, Levine JE, Reddy P, Holler E. Graft-versus-host disease. Lancet. 2009;373:1550-61.

11. Aslanian H, Chander B, Robert M, et al. Prospective evaluation of acute graft-versus-host disease. Dig Dis Sci. 2012;57:720-5.

12. Johansson JE, Nilsson O, Stotzer PO. Colonoscopy and sigmoidoscopy are equally effective for the diagnosis of colonic acute graft-versus-host disease in patients with diarrhea after allogeneic stem cell transplantation: A prospective controlled trial. Biol Blood Marrow Transpl. 2015;21(12):2086-90.

13. Domiati-saad R, Klintmalm GB, Netto G, Agura ED, Chinnakotla S, Smith DM. Acute graft versus host disease after liver transplantation: Patterns of lymphocyte chimerism. Am J Transpl. 2005;5(12):2968-73.

14. Star KV, Ho VT, Wang HH, Odze RD. Histologic features in colon biopsies can discriminate mycophenolate from GVHD-induced colitis. Am J Surg Pathol. 2013;37(9):1319-28.

15. Salomao M, Dorritie K, Mapara MY, Sepulveda A. Histopathology of graftvs-host disease of gastrointestinal tract and liver: An update. Am J Clin Pathol. 2016;145(5):591-603.

16. Nepal S, Navaneethan U, Bennett AE, Shen B. De novo inflammatory bowel disease and its mimics after organ transplantation. Inflamm Bowel Dis. 2013;19:1518-27.

17. Bertz H, Afting M, Kreisel W, Duffner U, Greinwald R, Finke J. Feasibility and response to budesonide as topical corticosteroid therapy for acute intestinal GVHD. Bone Marrow Transpl. 1999;24:1185-9.

18. Houston BL, Yan M, Tinckam K, et al. Extracorporeal photopheresis in solid organ transplant-associated acute graft-versus-host disease. Transfusion. 2016;56(4):962-9.

Copyright: ( 2019 The Author(s). Published by Wolters Kluwer Health, Inc. on behalf of The American College of Gastroenterology. This is an open-access article distributed under the terms of the Creative Commons Attribution-Non Commercial-No Derivatives License 4.0 (CCBY-NC-ND), where it is permissible to download and share the work provided it is properly cited. The work cannot be changed in any way or used commercially without permission from the journal. 